\title{
SOME EXPANSION FORMULAS FOR A CLASS OF SINGULAR PARTIAL DIFFERENTIAL EQUATIONS
}

\author{
ABDULLAH ALTIN
}

\begin{abstract}
We obtain a generalization of the Almansi's expansion and the Lord Kelvin principle for solutions of a class of iterated elliptic or ultrahyperbolic equations. We also obtain some homogeneous function expansions for solutions of the same equations.
\end{abstract}

1. Introduction. In this paper we present some expansion formulas for solutions of a class of iterated elliptic or ultrahyperbolic partial differential equations. Specifically, we consider the elliptic or ultrahyperbolic equation

$$
L u=\sum_{i=1}^{n}\left(\frac{\partial^{2} u}{\partial x_{i}^{2}}+\frac{\alpha_{i}}{x_{i}} \frac{\partial u}{\partial x_{i}}\right) \pm \sum_{i=1}^{s}\left(\frac{\partial^{2} u}{\partial y_{i}^{2}}+\frac{\beta_{i}}{y_{i}} \frac{\partial u}{\partial y_{i}}\right)+\frac{\gamma u}{r^{2}}=0
$$

where the constants $\alpha_{1}, \ldots, \alpha_{n}, \beta_{1}, \ldots, \beta_{s}$ and $\gamma$ are real parameters and

$$
r^{2}=\sum_{i=1}^{n} x_{i}^{2} \pm \sum_{i=1}^{s} y_{i}^{2}=x^{2} \pm y^{2} .
$$

The domain of the operator $L$ is the set of all real-valued functions $u(x, y)$ of class $C^{2}(D)$ where $x=\left(x_{1}, \ldots, x_{n}\right)$ and $y=\left(y_{1}, \ldots, y_{s}\right)$ denote points in $R^{n}$ and $R^{s}$, respectively, and $D$ is a regularity domain of $u$ in $R^{n+s}$. Clearly the equation (1) includes some of the well-known classical equations such as the Laplace equation, the wave equation and the EPD and GASPT equations [1-9].

2. Expansion formulas. We first establish some properties of the operator $L$. From the definition of $L$, it can be shown by induction on $p$ that

$$
L^{p}\left(r^{m}\right)=\prod_{j=0}^{p-1}[(m-2 j)(m-2 j+\phi)+\gamma] r^{m-2 p}
$$

where $m$ is a real parameter and

$$
\phi=n+s-2+\sum_{i=1}^{n} \alpha_{i}+\sum_{i=1}^{s} \beta_{i}
$$

Received by the editors July 7, 1981.

1980 Mathematics Subject Classification. Primary 35C05.

Key words and phrases. Iterated equation, elliptic and ultrahyperbolic equations, Almansi's expansion, Lord Kelvin principle, homogeneous functions, induction method. 
Let $u, v \in C^{2}(D)$ be any two functions. By direct calculation we see that

$$
L(u v)=u L(v)+v L(u)-\frac{\gamma}{r^{2}} u v+2\left(\sum_{i=1}^{n} \frac{\partial u}{\partial x_{i}} \frac{\partial v}{\partial x_{i}} \pm \sum_{i=1}^{s} \frac{\partial u}{\partial y_{i}} \frac{\partial v}{\partial y_{i}}\right) .
$$

Hence if $u \in C^{2}(D)$ is a solution of the equation $L u=0$, then by (3) and (5),

$$
L\left(r^{m} u\right)=r^{m-2} m\left(m+\phi+2 T^{*}\right) u
$$

where

$$
T^{*}=\sum_{i=1}^{n} x_{i} \frac{\partial}{\partial x_{i}}+\sum_{i=1}^{s} y_{i} \frac{\partial}{\partial y_{i}} .
$$

By direct calculation, it can be shown that

$$
L T^{*}=\left(2+T^{*}\right) L .
$$

Hence if we set $T=m+\phi+2 T^{*}$, then from (7) we get

$$
L T=(4+T) L \text {. }
$$

LEMMA 1. Let $u$ be any solution of the equation $L u=0$. Then, for any positive integer $p$ and for any real number $m$,

$$
L^{p}\left(r^{m} u\right)=r^{m-2 p} \prod_{j=0}^{p-1}(m-2 j)\left(m-2 j+\phi+2 T^{*}\right) u .
$$

Proof. From (6) we have $L\left(r^{m} u\right)=m r^{m-2} T u$. Applying the operator $L$ on both sides of this equality and using (5) and (8) we have

$$
\begin{aligned}
L^{2}\left(r^{m} u\right) & =m L\left(r^{m-2} T u\right)=m r^{m-2}(4+T) L u+r^{m-4} m(m-2)(-2+T) T u \\
& =r^{m-4} m(m-2) T(-2+T) u \\
& =r^{m-4} m(m-2)\left(m+\phi+2 T^{*}\right)\left(m-2+\phi+2 T^{*}\right) u .
\end{aligned}
$$

Hence by induction we obtain the formula (9).

By making use of Lemma 1 we shall now give two expansion formulas.

(a) A generalization of the Almansi's expansion. Let $u_{j}(x, y), j=0,1, \ldots, p-1$, be any $p$ solutions of the equation $L u=0$. Then by Lemma 1

$$
L^{p}\left[r^{2 j} u_{j}(x, y)\right]=0 \text { for } j=0,1, \ldots, p-1 .
$$

Thus each of the functions $r^{2 j} u_{j}(x, y)$ and therefore their superposition

$$
w=\sum_{j=0}^{p-1} r^{2 j} u_{j}(x, y)
$$

satisfies the equation $L^{p} w=0$. This is the analogue of the Almansi expansion given for the Laplace operator [1]. Hence we call the representation (10) an Almansi's expansion.

(b) A homogeneous function expansion. Let the function $u(x, y)$ be a homogeneous solution of degree $\lambda$, of the equation $L u=0$. Then, by the Euler's theorem, we know that

$$
T^{*} u(x, y)=\sum_{i=1}^{n} x_{i} \frac{\partial u}{\partial x_{i}}+\sum_{i=1}^{s} y_{i} \frac{\partial u}{\partial y_{i}}=\lambda u(x, y)
$$


Hence by (9) we have

$$
L^{p}\left(r^{m} u\right)=r^{m-2 p} \prod_{j=0}^{p-1}(m-2 j)(m-2 j+\phi+2 \lambda) u .
$$

This shows that if the functions $u_{j}, j=0,1, \ldots, p-1$ are homogeneous solutions of degree $\lambda_{j}$ of the equation $L u=0$ then, for each of the values $m=2 j-\phi-2 \lambda_{j}$, $j=0,1, \ldots, p-1$, the functions

$$
r^{2 j-\phi-2 \lambda_{i}} u_{j}(x, y), \quad j=0,1, \ldots, p-1,
$$

satisfy the equation $L^{p} u=0$. It follows that the linear combination

$$
w=\sum_{j=0}^{p-1} r^{2 j-\phi-2 \lambda_{i}} u_{j}(x, y)
$$

is also a solution. Here we noted that the operators on the right of (9) are commutative.

3. Generalized Lord Kelvin principle. The classical Kelvin principle was given for harmonic functions in 1847 [5]. Germain and Bader [3] stated this principle in two-dimensional space for the Tricomi equation in 1953. Their result was extended by A. Huber [4] for the equation

$$
\sum_{i=1}^{n} \frac{\partial^{2} u}{\partial x_{i}^{2}}+\frac{\partial^{2} u}{\partial y^{2}}+\frac{k}{y} \frac{\partial u}{\partial y}=0, \quad-\infty<k<\infty,
$$

in 1956. A generalization of Kelvin principle was established by A. Weinstein [9] in 1960 by the use of polar coordinates for the equation

$$
\sum_{i=1}^{n}\left(\frac{\partial^{2} u}{\partial x_{i}^{2}}+\frac{k_{i}}{x_{i}} \frac{\partial u}{\partial x_{i}}\right)=0, \quad-\infty<k_{i}<\infty .
$$

Weinstein's result was obtained again by A. O. Celebi [2] in 1968 by using rectangular coordinates.

To establish the Lord Kelvin principle for solutions of (1), let $\xi_{i}=x_{i} / r^{2}$, $i=1, \ldots, n, \eta_{i}=y_{i} / r^{2}, i=1, \ldots, s$. Then, by using the same method as in [2] and [4] we can show that

$$
L\left[r^{m} u(\xi, \eta)\right]=r^{m-2}(m+\phi)\left(m-2 T_{1}^{*}\right) u(\xi, \eta)+r^{m-4} L_{(\xi, \eta)}[u(\xi, \eta)]
$$

where $L_{(\xi, \eta)}$ denotes the operator $L$ with $x$ and $y$ replaced by $\xi$ and $\eta$, respectively, $\phi$ is defined in (4), $\xi=\left(\xi_{1}, \ldots, \xi_{n}\right), \eta=\left(\eta_{1}, \ldots, \eta_{s}\right)$ and

$$
T_{1}^{*}=\sum_{i=1}^{n} \xi_{i} \frac{\partial}{\partial \xi_{i}}+\sum_{i=1}^{s} \eta_{i} \frac{\partial}{\partial \eta_{i}} .
$$

Thus if $u(x, y)$ is a solution of $L u=0$, then it follows from (12) that $L\left[r^{-\phi} u(\xi, \eta)\right]$ $=0$. That is, the function $v=r^{-\phi} u(\xi, \eta)$ or, explicitly,

$$
v=r^{2-n-s-\sum_{i=1}^{n} \alpha_{i}-\sum_{i=1}^{s} \beta_{i}} u\left(\frac{x_{1}}{r^{2}}, \ldots, \frac{x_{n}}{r^{2}}, \frac{y_{1}}{r^{2}}, \ldots, \frac{y_{s}}{r^{2}}\right)
$$

is also a solution of the same equation. This establishes the Kelvin principle for the equation (1). 
By making use of the formula (12) we shall obtain further results.

If $u(x, y)$ is a homogeneous solution of degree $\lambda$ of the equation (1), then

$$
T_{1}^{*} u(\xi, \eta)=\sum_{i=1}^{n} \xi_{i} \frac{\partial u}{\partial \xi_{i}}+\sum_{i=1}^{s} \eta_{i} \frac{\partial u}{\partial \eta_{i}}=\lambda u(\xi, \eta) .
$$

Hence, by the formula (12) it is clear that $L\left[r^{2 \lambda} u(\xi, \eta)\right]=0$, that is, the function $v=r^{2 \lambda} u(\xi, \eta)$ is a solution of the equation (1).

Let $u(x, y)$ be a solution of (1). Then (12) becomes

$$
L\left[r^{m} u(\xi, \eta)\right]=r^{m-2}(m+\phi)\left(m-2 T_{1}^{*}\right) u(\xi, \eta) .
$$

Hence as in (7) it is clear that for $L_{(\xi, \eta)}$ and $T_{1}^{*}$ we also have

$$
L_{(\xi, \eta)} T_{1}^{*}=\left(2+T_{1}^{*}\right) L_{(\xi, \eta)} \text {. }
$$

Thus by induction we obtain

$$
L^{p}\left[r^{m} u(\xi, \eta)\right]=r^{m-2 p} \prod_{j=0}^{p-1}(m-2 j+\phi)\left(m-2 j-2 T_{1}^{*}\right) u(\xi,-\eta) .
$$

Now by using (14), we can establish the following two expansion formulas which correspond to those given in $\$ 2$.

(a) Let $u_{j}(x, y), j=0,1, \ldots, p-1$, be any $p$ solutions of the equation $L u=0$; then by (14) it follows that

$$
L^{p}\left[r^{2 j-\phi} u_{j}(\xi, \eta)\right]=0 \quad \text { for } j=0,1, \ldots, p-1 .
$$

Thus each of the functions $r^{2 j-\phi} u_{j}(\xi, \eta)$ and therefore their superposition

$$
w=\sum_{j=0}^{p-1} r^{2 j-\phi} u_{j}(\xi, \eta)
$$

satisfies the equation $L^{p} w=0$.

(b) Let the function $u(x, y)$ be a homogeneous solution of degree $\lambda$, of the equation $L u=0$. Then by the Euler theorem, we know that

$$
T_{1}^{*} u(\xi, \eta)=\sum_{i=1}^{n} \xi_{i} \frac{\partial u}{\partial \xi_{i}}+\sum_{i=1}^{s} \eta_{i} \frac{\partial u}{\partial \eta_{i}}=\lambda u(\xi, \eta) .
$$

Hence by (14) we have

$$
L^{p}\left[r^{m} u(\xi, \eta)\right]=r^{m-2 p} \prod_{j=0}^{p-1}(m-2 j+\phi)(m-2 j-2 \lambda) u(\xi, \eta) .
$$

This shows that if the functions $u_{j}(x, y), j=0,1, \ldots, p-1$, are homogeneous solutions of degree $\lambda_{j}$ of the equation $L u=0$, then for each of the values $m=2 j+$ $2 \lambda_{j}, j=0,1, \ldots, p-1$, the functions $r^{2\left(j+\lambda_{j}\right)} u_{j}(\xi, \eta), j=0,1, \ldots, p-1$, satisfy the equation $L^{p} u=0$. It follows that the linear combination

$$
w=\sum_{j=0}^{p-1} r^{2\left(j+\lambda_{j}\right)} u_{j}(\xi, \eta)
$$

is also a solution. 


\section{REFERENCES}

1. E. Almansi, Sull' integrazione dell' differenziale $\Delta^{2 m} u=0$, Ann. Mat. Ser. II, III (1899), 1-59.

2. A. O. Celebi, On the generalized Tricomi's equation, Comm. Fac. Sci. Univ. Ankara Sér. A 17 (1968), $1-31$.

3. P. Germain and R. Bader, Sur le problème de Tricomi, Rend. Circ. Mat. Palermo (2) 2 (1953), 53-70.

4. A. Huber, Some results on generalized axially symmetric potential theory, Proc. Conf. on Partial Differential Equation, (Univ. of Maryland, 1956), pp. 147-155.

5. W. Thomson, Extraits de deux lettres adressées a M. Liouville, J. Math. Pures Appl. 12 (1847), 256.

6. D. Krahn, On the iterated wave equation, Nederl. Akad. Wetensch. Proc. Ser. A 60 (1957), 492-505.

7. L. E. Payne and W. Pell, The Stokes flow problem for a class of axially symmetric bodies, J. Fluid Mech. 7 (1960), 529-549.

8. A. Weinstein, On a class of partial differential equations of even order, Ann. Mat. Pura Appl. (4) 39 (1955), 245-254.

9. __ On a singular differential operator, Ann. Mat. Pura Appl. (4) 49 (1960), 359-365.

Faculty of Science, University of ANKara, Besevler, ANKara, Turkey 